\title{
Grain size refinement of Copper DHP by Solid State Processing
}

\author{
F. Simões****, R.M. Leal ${ }^{* * * * * *}$ and D.M. Rodrigues** \\ *ISEC - Mechanical Engineering Department, Engineering Institute of Coimbra, Portugal \\ **CEMUC - Department of Mechanical Engineering, University of Coimbra, Portugal. \\ ***ESAD.CR-IPL Caldas da Rainha, Portugal
}

Due to its good thermal and electrical conductivity, improved plastic properties and excellent corrosion and oxidation resistance, copper is being widely used worldwide. Nevertheless, for some particular applications [1, 2], it is still desirable to improve copper strength, wear and fatigue resistance properties. These characteristics can be enhanced by suitable modification of materials microstructure by using solid state processing techniques, such as Friction Stir Processing (FSP) [3]. This technique makes use of non-consumable rotating tools to induce heat and severe plastic deformation in the materials to be processed. The complex thermo-mechanical phenomena developed inside the stirred volume, promotes strong microstructural modifications, which cautiously controlled, enables deeply transforming the microstructure of the materials being processed. In fact, FSP thermo-mechanisms leads to significant grain refinement, which drove the use of this technique towards producing ultrafine grained structural metallic materials from aluminium and other ductile metallic alloys [3]. Due to the high macro and microstructural heterogeneity resulting from FSP methodologies, as well as the extremely refined structure resulting from it, the microstructural characterization of processed materials is an especially difficult task, which requires skilled researchers as well as important microscopy and microanalysis resources.

In present work, $1 \mathrm{~mm}$ thick plates of phosphorus deoxidised copper (Cu-DHP), temper class $\mathrm{R} 240$, were friction stir processed using the conical and scrolled shoulder tools, shown in Figure 1, and varying processing parameters, namely, two different tool rotation rates $(\omega=400$ and $1000 \mathrm{rpm})$ and a constant traverse speed $(v)$ of $250 \mathrm{~mm} / \mathrm{min}$. The specimens for analysis were removed transverse to the processing direction, and prepared for both optical and TEM analysis. The optical micrograph images were obtained from polished specimens, etched with $\mathrm{FeCl}_{3}$ and Hid reagents. Samples for TEM were polished mechanically from both sides down to a thickness of about $0.15 \mathrm{~mm}$ and then electropolished using a double jet thinner. A dilute solution of orthophosphoric acid $(2: 1)$ at room temperature under $10 \mathrm{~V}$ tension was used. TEM analysis was carried out at $100 \mathrm{kV}$.

A strong influence of the processing conditions on the microstructural refinement was observed, being found that the tool geometry, which has a close relation with the plastic deformation and dynamic recrystallization kinetics, inside the stirred volume, is determinant in FSP results. In Figures 2.a and b are shown, respectively, optical and TEM images of the non-processed material, showing a coarse grain structure with mean grain size of $18 \mu \mathrm{m}$. Most of the grains display families of parallel slip lines, as shown in the TEM image of Figure 2.b. In the same way, microstructural analysis of the processed samples revealed the formation of more or less refined grain structures, according to the processing conditions in use. In Figures 3 and 4 are shown pictures of processed samples, obtained using the conical and scrolled shoulder tools under hot $(\omega=1000 \mathrm{rpm})$ and cold $(\omega=400$ rpm) processing conditions. For the samples processed with the conical tool and hot processing conditions (Figure 3.a), the average grain size $(8 \mu \mathrm{m})$ was clearly coarser than that of the sample processed at $400 \mathrm{rpm}$ (Figure 3.b), for which the average grain size registered was $1 \mu \mathrm{m}$. For the samples processed with the scrolled tool (Figure 4) no important differences in grain size were obtained when changing the tool rotation rate. A significant decrease in grain size, up to $500 \mathrm{~nm}$, was registered for these samples. By comparing the results it is possible to conclude that, under similar processing conditions, the scrolled tool induced higher grain refinement than the conical tool, which is related with the stronger plastic deformation achievable with this geometry.

\section{References}

1. Surekha K. and Els-Botes A., Mater. Des., 32:911-916, 2011.

2. Barmouz M. et al., J. Mater. Charact., 62:108-117, 2011.

3. Mishra R.S. et al., Mater. Sci. Eng. A, 341:307-310, 2003. 

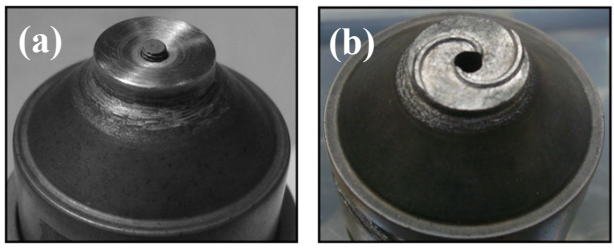

Figure 1. Conical (a) and scrolled shoulder (b) FSP tools
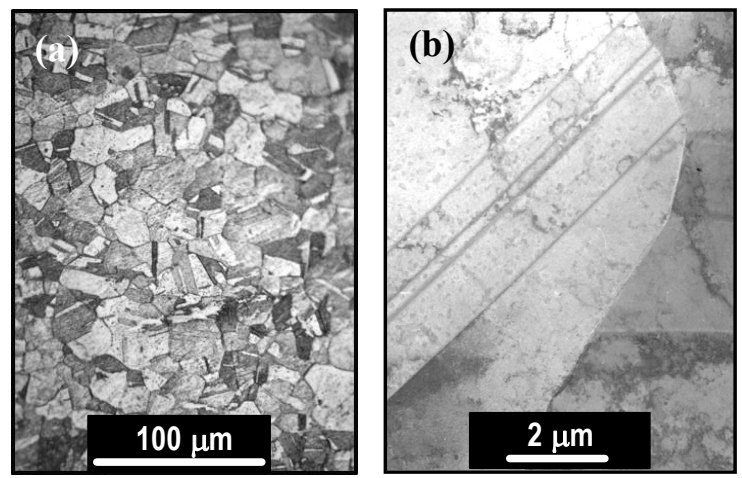

Figure2. (a) Optical micrograph image (500x) and (b) TEM image of the base material.
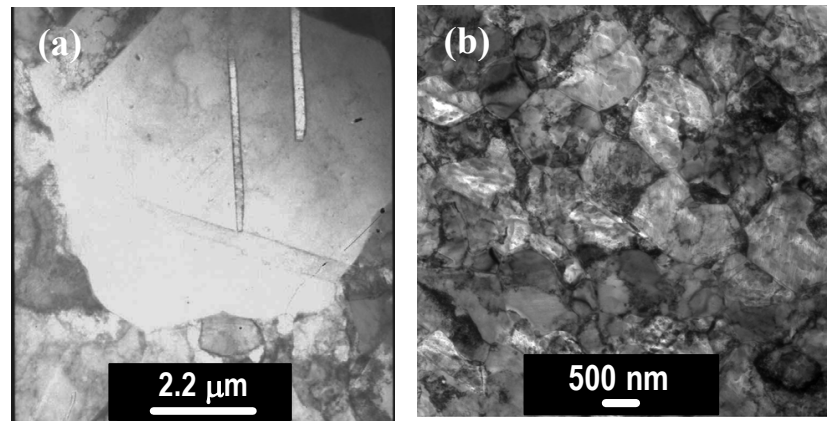

Figure 3. TEM images of copper processed with the conical tool at $1000 \mathrm{rpm}$ (a) and $400 \mathrm{rpm}$ (b).
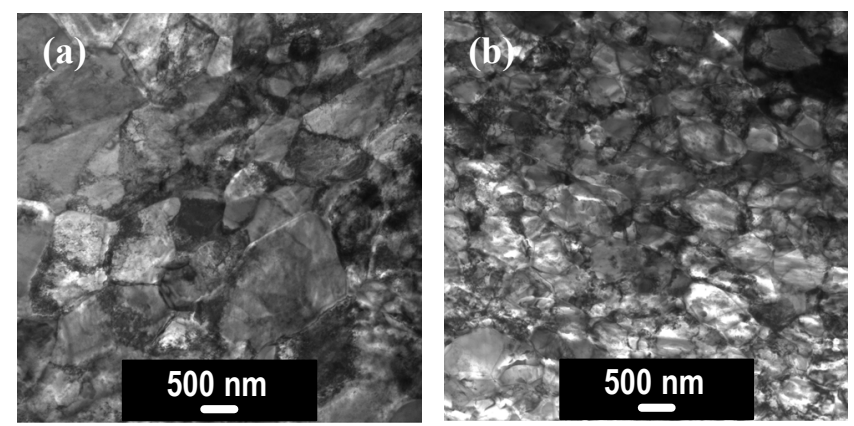

Figure 4. TEM images of copper processed with the scrolled tool at $1000 \mathrm{rpm}$ (a) and $400 \mathrm{rpm}$ (b). 